\title{
DISTRIBUTION OF VALUES OF ENTIRE FUNCTIONS OF LOWER ORDER LESS THAN ONE
}

\author{
By TADASHI KOBAYASHI
}

1. Introduction. Quite recently, Tsuzuki [3] has proved the following result :

Let $f(z)$ be an entire function of order less than one and let $\left\{w_{n}\right\}$ be an unbounded sequence. Assume that there exists $\beta$ such that $0<\beta<\pi / 2$ and all the roots of equations

belong to the sector

$$
f(z)=w_{n} \quad(n=1,2, \cdots),
$$

$$
\{z|| \arg z-\pi \mid \leqq \beta\} .
$$

Then $f(z)$ is a linear function.

The purpose of this paper is to generalize the above result by an elementary argument. The proof given here is quite different from that of Tsuzuki and, I hope, somewhat simpler.

THEOREM. Let $f(z)$ be an entire function and let $T(r, f)$ be its characteristic function. Assume that there exists an unbounded sequence $\left\{w_{n}\right\}$ such that all the roots of equations

lie in the half plane

$$
f(z)=w_{n} \quad(n=1,2, \cdots),
$$

Assume further that

$$
\left\{z|| \arg z-\pi \mid \leqq \frac{\pi}{2}\right\}
$$

$$
\liminf _{r \rightarrow \infty} \frac{T(r, f)}{r}=0 .
$$

Then $f(z)$ is a polynomial of degree not greater than two.

Considering Mittag-Leffler's function, we can easily assure that this theorem is no longer true when the opening of the sector is greater than $\pi$.

Further the assumption $\left(^{*}\right)$ cannot be improved, in general. This is easily seen on an example such that

$$
f(z)=\exp (-z)
$$

Received June 3, 1975. 
2. Preliminaries. Before proceeding with the proof of Theorem, we need some preliminary facts.

LEMMA 1. Let $f(z)$ be a nonconstant entire function satisfying the assumption $(*)$. If all the zeros $\left\{a_{n}\right\}$ of $f(z)$ are

$$
\operatorname{Re} a_{n} \leqq 0 \quad(n=1,2, \cdots),
$$

then

$$
\operatorname{Re} \frac{f^{\prime}(z)}{f(z)}>0
$$

in the right half plane

$$
R=\{z \mid \operatorname{Re} z>0\} .
$$

Proof. For each point $c$ in $R$, set

$$
f_{c}(z)=f(z+c) .
$$

Then it follows from an elementary formula [2] that

$$
\begin{aligned}
r \operatorname{Re} \frac{f_{c}^{\prime}(0)}{f_{c}(0)}= & \frac{1}{\pi} \int_{0}^{2 \pi} \log \left|f_{c}\left(r e^{i t}\right)\right| \cos t d t \\
& +\sum_{\left|a_{n}-c\right|<r} \operatorname{Re}\left(\frac{\bar{a}_{n}-\bar{c}}{r}-\frac{r}{a_{n}-c}\right)
\end{aligned}
$$

for every positive $r$. Hence from (1), we have

$$
\operatorname{Re} \frac{f_{c}^{\prime}(0)}{f_{c}(0)}=\operatorname{Re} \frac{f^{\prime}(c)}{f(c)} \geqq-4 \frac{T\left(r, f_{c}\right)}{r} .
$$

Therefore, since the assumption $\left(^{*}\right)$ also gives

$$
\liminf _{r \rightarrow \infty} \frac{T\left(r, f_{c}\right)}{r}=0,
$$

we conclude that

$$
\operatorname{Re} \frac{f^{\prime}(z)}{f(z)} \geqq 0
$$

for each point $z$ in $R$.

Here, notice that $\operatorname{Re}\left(f^{\prime}(z) / f(z)\right)$ is harmonic in $R$. Then we obtain (2) excepting when

$$
f(z)=\exp (a z+b) .
$$

This completes the proof of Lemma 1.

Let $f(z)$ be a nonconstant entire function satisfying the hypotheses of Theorem. Then by Lemma 1 ,

$$
\operatorname{Re} \frac{f^{\prime}(z)}{f(z)-w_{n}}>0 \quad(n=1,2, \cdots),
$$

in the right half plane $R$. In particular, the first derivative $f^{\prime}(z)$ has no zeros 
there.

Now we consider the argument of $f^{\prime}(z)$ which is denoted by $u(z)$. Let us set

$$
\gamma_{n}=\arg w_{n} \quad(n=1,2, \cdots) .
$$

Then the inequalities (3) will be

so that

$$
\operatorname{Re} \frac{f(z)}{f^{\prime}(z)}>\left|\frac{w_{n}}{f^{\prime}(z)}\right| \cos \left(u(z)-\gamma_{n}\right)
$$

$$
|f(z)|>\left|w_{n}\right| \cos \left(u(z)-\gamma_{n}\right) \quad(n=1,2, \cdots),
$$

for every point $z$ in $R$. These inequalities (4) are essential to our proof.

Here we assume that there exist four points $a, b, c$ and $d$ in the right half plane $R$ such that

$$
u(a)=u(c)-\pi
$$

and

$$
u(a)<u(b)<u(c)<u(d)<u(a)+2 \pi .
$$

Then it is possible to find a positive number $\varepsilon$ such that

$$
\begin{aligned}
& u(a)+\varepsilon<u(b)<u(c)-\varepsilon, \\
& u(c)+\varepsilon<u(d)<u(a)+2 \pi-\varepsilon .
\end{aligned}
$$

According to the inequalities (4), for each $n(n=1,2, \cdots)$,

$$
\begin{aligned}
& |f(a)|>\left|w_{n}\right| \cos \left(u(a)-\gamma_{n}\right), \\
& |f(c)|>\left|w_{n}\right| \cos \left(u(c)-\gamma_{n}\right) .
\end{aligned}
$$

Therefore, since the sequence $\left\{w_{n}\right\}$ is unbounded, infinitely many terms of $\left\{w_{n}\right\}$ must satisfy

$$
\pi-\varepsilon \leqq 2\left|\gamma_{n}-u(a)\right| \leqq \pi+\varepsilon .
$$

But this clearly contradicts (4) and (7). Hence we cannot take four points in $R$ satisfying (5) and (6). By this fact, we easily have the following lemma.

LEMMA 2. Let $f(z)$ be a nonconstant entire function satisfying the hypotheses of Theorem. Then it is possible to find a real number $\gamma$ such that

$$
\left|\arg f^{\prime}(z)-\gamma\right| \leqq \frac{\pi}{2}
$$

for every point $z$ in the right half plane $R$.

3. Proof of Theorem. We may assume that $f(z)$ is not linear. Then by Lemma 2, there exists a real number $\gamma$ such that 


$$
\left|\arg f^{\prime}(z)-\gamma\right| \leqq \frac{\pi}{2}
$$

for every point $z$ in $R$. Set

(9)

$$
v_{2 n-1}=n \exp \left(i \gamma+i \frac{2}{3}-\pi\right) \text {, }
$$

$$
v_{2 n}=n \exp \left(\imath \gamma-i \frac{2}{3}-\pi\right) \quad(n=1,2, \cdots) .
$$

Then it follows from (8) that all the roots of equations

$$
f^{\prime}(z)=v_{n} \quad(n=1,2, \cdots),
$$

belong to the half plane

$$
\{z \mid \operatorname{Re} z \leqq 0\} \text {. }
$$

Further by an elementary estimation, we also have

$$
\liminf _{r \rightarrow \infty} \frac{T\left(r, f^{\prime}(z)\right)}{r}=0 .
$$

Hence by the same argument which is developed in the section 2 , the second derivative $f^{\prime \prime}(z)$ has no zeros in the right half plane $R$ and

$$
\operatorname{Re}-\frac{f^{\prime}(z)}{f^{\prime \prime}(\bar{z})}>\operatorname{Re} \frac{v_{n}}{f^{\prime \prime}(z)} \quad(n=1,2, \cdots),
$$

there. Thus from the definition (9) of the sequence $\left\{v_{n}\right\}$ and the inequalities (10), we obtain

$$
\left|\arg f^{\prime \prime}(z)-\gamma\right| \leqq \frac{\pi}{6}
$$

for each point $z$ in $R$. Therefore by (11), using the same argument once more, we easily conclude that

which yields the desired result.

$$
f^{\prime \prime}(z)=C,
$$

4. Remarks. Finally, it might be of interest to mention that our Lemma 1 is sufficient to yield the following facts which are analogues of Lucas' theorem [1].

(I) Let $f(z)$ be a nonconstant entire function satisfying

$$
\liminf _{r \rightarrow \infty} \frac{T(r, f)}{r}=0 .
$$

Then the smallest convex set which contains the zeros of $f(z)$ also contains the zeros of $f^{\prime}(z)$.

(II) Let $f(z)$ be a nonconstant entire function which satisfies 


$$
\liminf _{r \rightarrow \infty} \frac{T(r, f)}{r}=0 \text {. }
$$

Then the smallest convex set which contains the zeros and ones of $f(z)$ also contains all the roots of equations

$$
f(z)=t \quad(0 \leqq t \leqq 1)
$$

REFERENCES

[1] Ahlfors, L.V., Complex analysis, McGraw-Hill, 1953.

[2] Hayman, W.K., Meromorphic functions, Oxford Univ. Press, 1964.

[3] Tsuzuki, M., On the value distribution of entire functions of order less than one, J. College of Liberal Arts, Saitama Univ. 9 (1974), 1-3.

Department of Mathematics,

Tokyo Institute of Technology. 